\title{
DKKI Positively Correlates with Lung Function in COPD Patients and Reduces Airway Inflammation
}

\author{
Luqi Dai ${ }^{1,2, *}$ \\ Dan $\mathrm{Xu}^{1, *}$ \\ Chun Wan ${ }^{1,2}$ \\ Lian Liu' \\ Fuqiang Wen $\mathbb{D}^{1,2}$ \\ 'Division of Pulmonary Diseases, State \\ Key Laboratory of Biotherapy, West \\ China Hospital, West China School of \\ Medicine, Sichuan University, Chengdu, \\ Sichuan, People's Republic of China; \\ ${ }^{2}$ Department of Respiratory and Critical \\ Care Medicine, West China Hospital, \\ West China School of Medicine, Sichuan \\ University, Chengdu, Sichuan, People's \\ Republic of China \\ *These authors contributed equally to \\ this work
}

\begin{abstract}
Purpose: WNT/ $\beta$-catenin signal pathway is a potential hope for lung tissue repair. We investigated the levels of Dickkopf-1 (DKK1), an endogenous inhibitor of WNT/ $\beta$-catenin signal pathway, in chronic obstructive pulmonary disease (COPD) patients and airway inflammation.
\end{abstract}

Patients and Methods: Collected the demographic and clinical characteristics of 36 healthy controls, 25 stable COPD patients and 10 acute exacerbation of COPD (AECOPD) patients, then performed pulmonary function and detected serum DKK1 levels. After overexpression of DKK1, detect the levels of DDK1, lipoprotein-related protein 6 (LRP6) and inflammatory factors in bronchial epithelial cells stimulated with cigarette smoke extract (CSE).

Results: Serum DKK1 were reduced in stable COPD patients compared to healthy controls $(3866.72 \pm 775.33 \mathrm{pg} / \mathrm{mL}$ vs $5317.61 \pm 1317.20 \mathrm{pg} / \mathrm{mL}, \mathrm{p}<0.0001)$, but there was no significant difference between stable and acutely exacerbated patients $(3866.72 \pm 775.33$ $\mathrm{pg} / \mathrm{mL}$ vs $3482.10 \pm 841.25 \mathrm{pg} / \mathrm{mL}, \mathrm{p}>0.05)$. DKK1 was positively correlated with FEV1 ( $\mathrm{r}$ $=0.570, \mathrm{p}<0.0001)$, FEV1/FVC $(r h o=0.590, \mathrm{p}<0.0001)$, FEV1/Pre $(\mathrm{r}=0.517, \mathrm{p}<0.0001)$. Multiple linear regression analysis also suggested that FEV1 levels were higher with increasing DKK1. In vitro, elevated IL-6, IL-8, TNF- $\alpha$ and decreased DKK1, LRP6 were found in Beas-2B cells after CSE treatments, and increased LRP6 and decreased inflammatory factors were found after overexpression of DKK1. Andrographolide restored the CSEinduced decrease in DKK1 and increase in IL-6 and IL-8.

Conclusion: DKK1 levels were decreased in COPD patients and positively correlated with lung function, overexpression of DKK1 and andrographolide attenuated airway cell inflammation, both suggesting a potential role in pathophysiology and providing a disease-specific biomarker pattern.

Keywords: LRP6, lung function, biomarker, andrographolide

\section{Introduction}

Dickkopf-1 (DKK1) a secretory glycoprotein, is an important member of DKK protein family. It was first discovered by Glinka and his colleagues in the embryonic cells of Xenopus in 1998. ${ }^{1}$ DKK protein family is an ancient and evolutionarily conserved gene family, which consists of 255-350 amino acid residues, including a signal peptide sequence and two conserved cysteine rich regions. ${ }^{2-4}$ The abnormal expression of DKK1 can affect the expression of certain proteins and genes in many signaling pathways. At present, the research on DKK1 focuses on the background of canonical WNT/ $\beta$-catenin signaling pathway, and DKK1 is recognized as a well-established inhibitor of $\beta$-catenin-dependent WNT signal. The C-terminal cysteine rich region of DKK1 can 
bind with low density lipoprotein-related protein 6 (LRP6) and kremen2, then blocking WNT signaling pathway. ${ }^{5-7}$

The WNT signaling pathway, which is active during the normal development of the lung, its abnormal activation is involved in the development of many chronic lung diseases including chronic obstructive pulmonary disease (COPD). ${ }^{8-10}$ Previous studies have found that WNT/ $\beta$-catenin signaling pathway activity at the lung tissue of COPD patients is reduced, while activation of this pathway can reduce cigarette smoke-induced lung inflammation and elastase-induced emphysema in mice. ${ }^{11-13}$ Studies have shown that circulating levels of DKK1 are associated with a variety of inflammatory diseases such as osteoarthritis, rheumatoid arthritis, periodontitis, and acute infections. ${ }^{14,15}$ As for the lung, DKK1 is involved in regulating WNT-induced epithelial cell proliferation in a dose-dependent manner. ${ }^{16}$ Mice lung inflammation is relieved after intratracheal administration of $\mathrm{Wnt} 3 \mathrm{a}$ or antibodies neutralizing DKK1. ${ }^{17}$

The above studies suggest that DKK1 may play a role in chronic inflammation in COPD, and in this study, we measured DKK1 in the blood circulation of COPD patients and healthy controls, then further explored the relationship between DKK1 levels and lung function.

\section{Materials and Methods}

\section{Patient Inclusion}

The research protocol followed the principles of the Declaration of Helsinki and was approved by the Institutional Review Board for Human Studies of West China Hospital of Sichuan University, China (Approved No. of ethic committee:2017(270)). During the period from January to October 2016 to December 2018, we randomly recruited healthy subjects, stable COPD patients and AECOPD patients from the outpatient and inpatient departments of West China Hospital. All subjects signed written informed consent. Pulmonary function in COPD patients met the diagnostic criteria of Global Strategy for the Diagnosis, Management, and Prevention of COPD (updated 2015). Based on the value of FEV1/FVC, COPD patients can be divided into 4 grades: GOLD stage I, $\geq 80 \%$; GOLD stage II, $\geq 50 \%$ to $<80 \%$; GOLD stage III, $\geq 30 \%$ to $<50 \%$; and GOLD stage IV, $<30 \%$ predicted). Subjects with lung-related disease (lung cancer, asthma, etc.), severe systemic disease, or medical history known to affect circulating DKK1 (including osteoarthritis, rheumatoid arthritis, acute infection and many other diseases) were excluded. ${ }^{14,15}$

\section{Collection of the Basic Information}

Information such as name, gender, ethnicity, age, height, weight, smoking history, symptoms, and lung function data of included subjects were collected. All subjects were tested for pulmonary function by a professional.

\section{Blood Collection and Assays}

All subjects were required to fast from $9 \mathrm{pm}$ the night before; the venous blood samples were collected the next morning, 1 hour later, centrifuged at $3000 \mathrm{rpm}$ for $10 \mathrm{~min}$ utes to separate the serum, and stored at $-80^{\circ} \mathrm{C}$ until analysis. DKK1, TNF- $\alpha$, and IL-6 were measured using Magnetic Luminex Screening Assay (R\&D Systems, Minneapolis, MN, USA) in the laboratory of West China Hospital.

\section{Cell Culture and Transfection}

The bronchial epithelial cell line BEAS-2B was purchased from ATCC and cultured in DMEM (Gibco Life Technologies) containing 10\% fetal bovine serum (Gemini, 900-108) and $1 \%$ penicillin and streptomycin, cigarette smoke extract was obtained from commercial cigarettes (Marlboro, Phillips Morris, Richmond, VA, USA; $1.0 \mathrm{mg}$ nicotine and $11 \mathrm{mg}$ tar per cigarette), CD513B-1 and pCD513B-1-DKK1 plasmids were purchased from PPL (Nanjing, China), lipofectamine 3000 (Invitrogen Life Technologies) was used to transfect the plasmid into BEAS2B cells.

\section{Western Blotting and ELISA Assay}

RIPA lysis buffer, protease inhibitor cocktail (beyotime, China) and PMSF (Cell Signaling Technology, Danvers, MA, USA) were added to the cells. After complete lysis, centrifuged at $12000 \mathrm{~g}$ for 30 minutes. The obtained protein was denatured with SDS loading buffer. Protein was fractionated by SDS polyacrylamide gel electrophoresis and incubated overnight at with antibodies against DKK1 (Affinity, China), GAPDH (ZENBIO, China), LRP6 (Cell Signaling Technology), respectively. After washing several times with TBST, the membranes were incubated with a secondary antibody. Collect the cell supernatant and use enzyme-linked immunosorbent assay (USA R\&D Systems) to detect IL-6, IL-8 and TNF- $\alpha$ according to the manufacturer's instructions. 


\section{Statistical Analysis}

All the data were analyzed by SPSS 22.0 for windows (IBM, Chicago, IL), and graphs were drawn with GraphPad Prism 6.01 for Windows (GraphPad Software Inc, La Jolla, CA, USA). Shapiro-Wilk test was used to determine whether quantitative data follow a normal distribution. If the data satisfied normal distribution, independent samples $t$ test, chi-square test and one-way ANOVA were used to compare differences between groups. Pearson's correlation coefficient and Spearman's rho were used to determine the correlation between DKK1 and lung function parameters in normal and non-normal data, respectively. All data were expressed as mean \pm standard deviation (SD). Bilateral $\mathrm{p}<0.05$ was considered statistically significant.

\section{Results}

COPD patients were defined as FEV 1: FVC ratio less than 0.7 after using bronchodilators. Our study ultimately included 71 subjects, including 36 controls, 25 patients with stable COPD, 10 patients with AECOPD. The baseline data are shown in Table 1 . FVC $(2.39 \pm 0.74 \mathrm{~L}$ vs $3.49 \pm 0.65$ L), FEV1 $(1.23 \pm 0.52 \mathrm{~L}$ vs $3.14 \pm 0.62 \mathrm{~L})$, FEV1/FVC $(51.13 \pm 12.12 \%$ vs $90.36 \pm 8.24 \%)$, FEV1/Pre $(51.28 \pm$ $19.14 \%$ vs $103.75 \pm 14.57 \%$ ) in stable COPD patients were significantly lower than those in the control group and this decrease was also observed in AECOPD patients $(\mathrm{p}<$ $0.0001)$. There was no significant difference in lung function between stable COPD and AECOPD patients $(\mathrm{p}>0.05)$.

\section{DKKI Decreased Significantly in COPD}

\section{Patients}

Compared with controls, serum DKK1 levels in patients with stable COPD and AECOPD were significantly reduced
$(5317.61 \pm 1317.20 \mathrm{pg} / \mathrm{mL}$ vs $3866.72 \pm 775.33 \mathrm{pg} / \mathrm{mL}$, $3482.10 \pm 841.25 \mathrm{pg} / \mathrm{mL}, \mathrm{p}<0.05$, Figure 1A). Since there were only 10 AECOPD patients, and the differences in baseline information and DKK1 levels between the two subgroups of COPD patients were not statistically significant, we performed a combined analysis. The results showed no significant difference in DKK1 levels between the smoking and nonsmoking groups ( $p>0.05$, Figure 1B). Similarly, due to the small sample size, individuals with GOLD stage I and II, individuals with GOLD stage III and IV, were combined respectively. GOLD stage III-IV patients serum showed greatest decrease in DKK1 compared to that of GOLD stage I-II patients and healthy subjects $(3428.88 \pm 726.46$ $\mathrm{pg} / \mathrm{mL}$ vs $4066.56 \pm 761.36 \mathrm{pg} / \mathrm{mL}, 5317.61 \pm 1317.20 \mathrm{pg} /$ $\mathrm{mL}, \mathrm{p}<0.05$, Figure $1 \mathrm{C}$ ).

\section{Correlation Between DKKI and Lung}

\section{Function}

Pulmonary function test is an important method for diagnosing and evaluating COPD patients, the correlation between DKK1 and lung function was analyzed. DKK1 was positively correlated with FVC $(r=0.462, p<0.0001)$, FEV1 $(r=0.570, \quad \mathrm{p}<0.0001), \quad$ FEV1/FVC $\quad(r h o=0.590$, $\mathrm{p}<0.0001$ ), and FEV1/Pre ( $\mathrm{rho}=0.517, \mathrm{p}<0.0001$, Table 2, Figure 2). After adjusting for age, gender, BMI, smoking history, the significant correlation was still observed in FEV1 $\quad(\mathrm{r}=0.358, \quad \mathrm{p}=0.003), \quad$ FEV1/FVC $\quad(\mathrm{r}=0.377$, $\mathrm{p}=0.002)$, FEV1/ Pre $(\mathrm{r}=0.346, \mathrm{p}=0.004)$, but no significant correlation was found between FVC and DKK1 $(\mathrm{r}=0.229, \mathrm{p}=0.063$, Table 2).

\section{Multiple Linear Regression Analysis}

In order to exclude other factors affecting DKK1, we conducted a multiple linear regression analysis of age,

Table I Characteristics of Healthy Controls, Stable COPD and AECOPD Patients

\begin{tabular}{|c|c|c|c|c|}
\hline Characteristics & $\begin{array}{l}\text { Healthy Controls } \\
(n=36)\end{array}$ & $\begin{array}{l}\text { Stable COPD Patients } \\
(n=25)\end{array}$ & $\begin{array}{l}\text { AECOPD Patients } \\
(n=10)\end{array}$ & $P$-value \\
\hline Age, years & $48.44 \pm 7.92$ & $64.20 \pm 8.09$ & $71.50 \pm 8.34$ & $<0.000 I^{*}$ \\
\hline Sex, male/female & $32 / 4$ & $20 / 5$ & $9 / 1$ & 0.570 \\
\hline BMI, kg/m² & $22.13 \pm 2.67$ & $23.26 \pm 3.29$ & $22.01 \pm 2.66$ & 0.281 \\
\hline Smoking history, Never-smoker/smoker, & $22 / 14$ & $7 / 18$ & $1 / 9$ & $0.003^{*}$ \\
\hline FVC, L & $3.49 \pm 0.65$ & $2.39 \pm 0.74$ & $2.05 \pm 0.69$ & $<0.000 I^{*}$ \\
\hline FEVI, L & $3.14 \pm 0.62$ & $1.23 \pm 0.52$ & $1.03 \pm 0.50$ & $<0.000 I^{*}$ \\
\hline FEVI/FVC, \% & $90.36 \pm 8.24$ & $51.13 \pm 12.12$ & $48.56 \pm 11.34$ & $<0.000 I^{*}$ \\
\hline FEVI/Pre, \% & $103.75 \pm 14.57$ & $51.28 \pm 19.14$ & $47.90 \pm 28.19$ & $<0.000 I^{*}$ \\
\hline DKKI, pg/mL & $5317.61 \pm 1317.20$ & $3866.72 \pm 775.33$ & $3482.10 \pm 841.25$ & $<0.000 I^{*}$ \\
\hline
\end{tabular}

Note: ${ }^{*} p<0.05$, the result is statistically significant. 
A

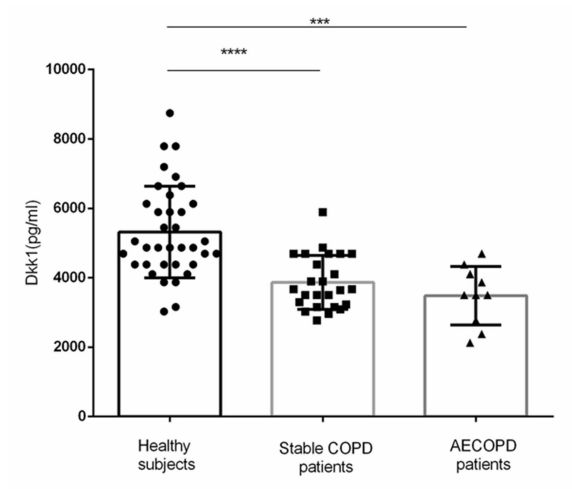

B

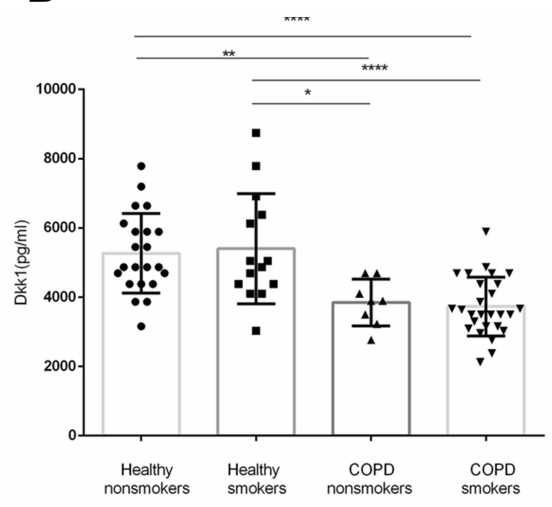

C

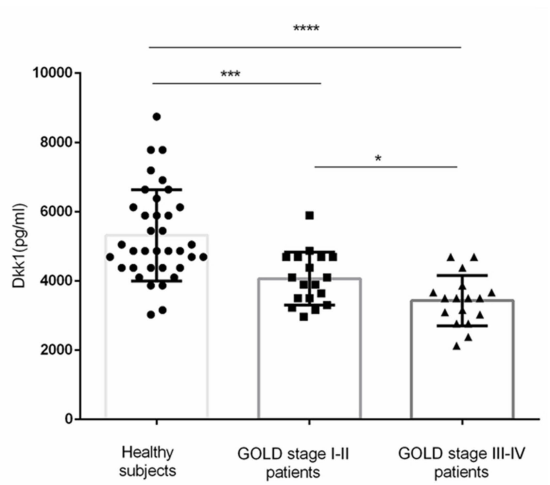

Figure I Levels of DKKI in serum.

Notes: $(A)$ The DKKI levels in stable COPD $(n=25)$ and AECOPD patients $(n=10)$ were significantly lower than those in healthy subjects $(n=36)$. $(B)$ No statistical difference between smokers and nonsmokers in healthy subjects or in COPD patients. (C) GOLD stage III-IV patient serum showed greatest decrease in DKKI compared to that of healthy subjects and GOLD stage I-II patients. DKKI, Dickkopf-I; GOLD=Global Initiative for Chronic Obstructive Lung Disease. * $p<0.05$, ** $p<0.0$ I, *** $<<0.00$ I, $* * * * p<0.0001$.

BMI, gender, FEV1, and FVC. The main results are shown in Table 3. The results showed no interaction between all subjects ( $\mathrm{DW}=2.089)$. These factors were independent of each other without collinearity $(\mathrm{VIF}<10)$ and could explain 39.8\% ( $\mathrm{R}$ square $=0.398)$ of the variance in DKK1. Both BMI and FEV1 significantly and positively affected the level of DKK1 $(\mathrm{B}=95.256, \mathrm{p}=0.038, \mathrm{~B}=$ $775.820, \mathrm{p}=0.016$, respectively).

\section{DKKI Regulates the Production of LRP6} and Inflammatory Factors in Response to CSE Exposure in BEAS-2B

Same as previous studies, CSE induced inflammation in airway epithelial cells. ${ }^{18,19}$ Enzyme-linked immunosorbent assay (ELISA) showed that TNF- $\alpha$ and IL-6 were upregulated in cells treated with different concentrations of CSE, and elevated IL-8 was observed in 3\% CSE and 4\% CSE-treated groups (Figure 3A). 2\% CSE, 3\% CSE and $4 \%$ CSE decreased the protein levels of DKK1 and

Table 2 Correlation Between DKKI and Lung Function

\begin{tabular}{|l|l|l|l|l|}
\hline \multirow{2}{*}{} & \multicolumn{2}{|l|}{ Linear Correlation } & \multicolumn{2}{l|}{ Partial Correlation } \\
\cline { 2 - 5 } & \multicolumn{2}{|l}{ DKKI } & \multicolumn{2}{l|}{ DKKI } \\
\cline { 2 - 5 } & r/rho & p & r & P \\
\hline FVC & 0.462 & $<0.000 I^{*}$ & 0.229 & 0.063 \\
FEVI & 0.570 & $<0.000 I^{*}$ & 0.358 & $0.003^{*}$ \\
FEVI/FVC & 0.590 & $<0.000 I^{*}$ & 0.377 & $0.002^{*}$ \\
FEVI/Pre & 0.517 & $<0.000 I^{*}$ & 0.346 & $0.004^{*}$ \\
\hline
\end{tabular}

Note: ${ }^{*} p<0.05$, the result is statistically significant.
LRP6 (Figure 3B). When DKK1 was overexpressed, CSE-stimulated LRP6 protein increased (Figure 3C) and TNF- $\alpha$ levels in the cell supernatant decreased (Figure 3D), suggesting that in epithelial cells, DKK1 can act as an upstream regulator of LRP6 in response to CSE stimulation and airway inflammation can be inhibited by DKK1. Pretreatment with andrographolide resulted in increased expression of LRP6 and DKK1 (Figure 3C) and decreased levels of IL-6 and IL-8 (Figure 3D).

\section{Discussion}

This study is the first to explore the role of DKK1 in COPD, bringing new implications to the role of DKK1 in the WNT pathway. DKK1 is recognized as an inhibitor of the canonical WNT/ $\beta$-catenin signaling pathway. ${ }^{6,20,21}$ Platelets are the main source of circulating DKK1, and an increase in DKK1 leads to the formation of leukocyteplatelet aggregates (LPA), which facilitates leukocyte infiltration. ${ }^{22}$ In the lung, DKK1 promotes macrophage and neutrophil infiltration by inhibiting the WNT pathway, and the infiltration of inflammatory cells is alleviated after inhibition of DKK $1 .{ }^{17}$ DKK1 can induce the production of IL-4, IL-10, IL-13 in CD4+ T cells and the production of Th2 cytokines, which together contribute to airway inflammation. $^{23}$ However, the relationship between DKK1 and COPD is still unclear. In this study, we compared the differences in circulating DKK1 in control subjects, patients with stable COPD and AECOPD, moreover, further analyzed the correlation between DKK1 and lung function. 

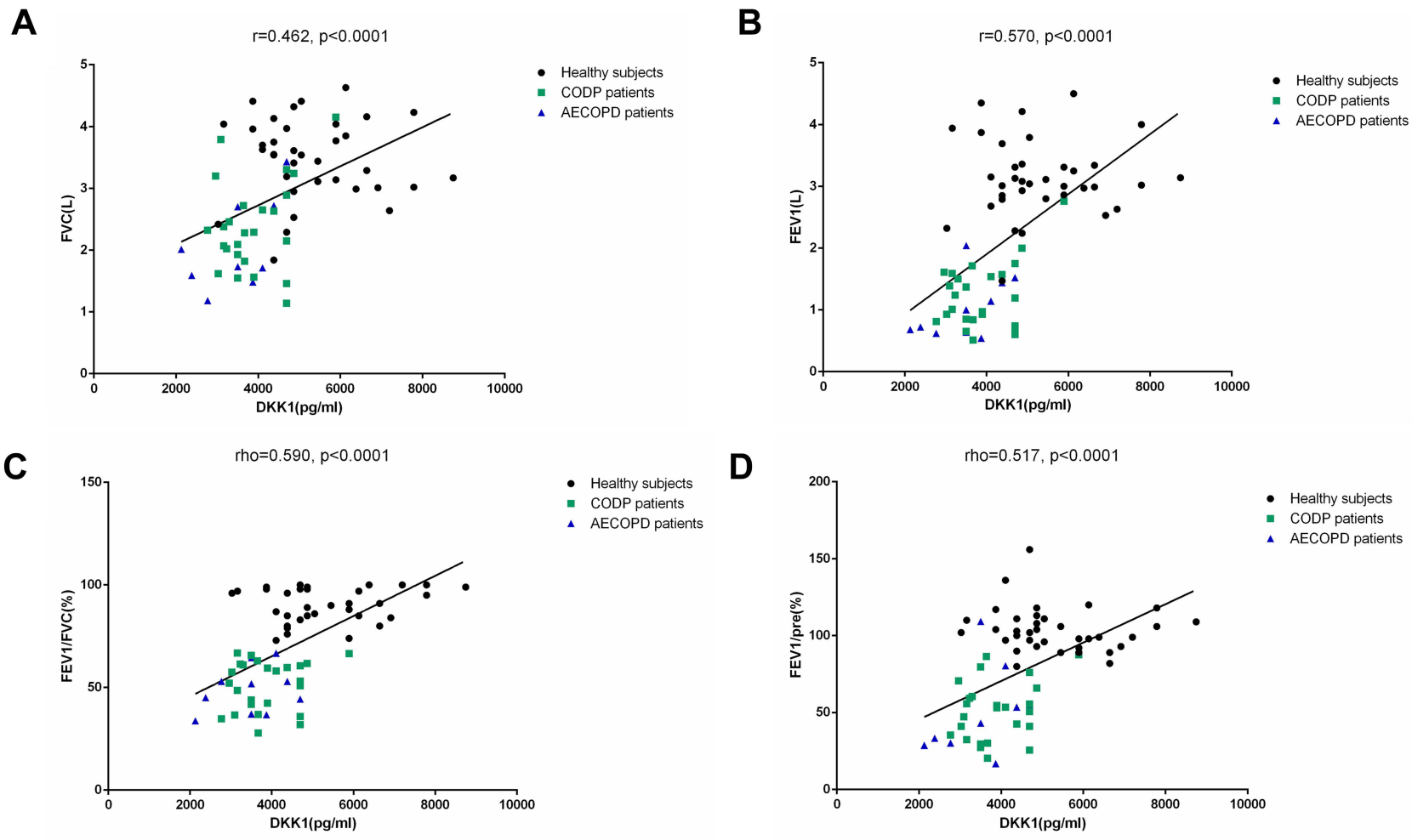

Figure 2 Correlation Between FVC, FEVI, FEVI/FVC, FEVI/Pre and DKKI.

Notes: DKKI levels were found to be positively correlated with (A) FVC ( $r=0.462, p<0.000 \mathrm{I}),(\mathbf{B}) \mathrm{FEVI}(\mathrm{r}=0.570, p<0.000 \mathrm{I}),(\mathbf{C}) \mathrm{FEVI} / \mathrm{FVC}$ (rho=0.590, $\mathrm{p}<0.000 \mathrm{I})$, and (D) FEVI (rho $=0.517, p<0.000 I$ ). Pearson's correlation coefficient $(r)$ and Spearman's rho were used to determine the correlation.

We found that DKK1 levels were decreased in both the stable COPD and AECOPD groups compared to the control group. The level of DKK1 in GOLD stage III-IV patients was lower than that of GOLD stage I-II. Correlation analysis showed that DKK1 was significantly positively correlated with FVC, FEV1, FEV1/FVC, and FEV1/Pre, additional multiple linear regression confirmed that with FEV1 significantly positively affect DKK1.These findings suggest that DKK1 is positively correlated with the lung function, the better the lung function, the higher the DKK1 level. Previous studies have shown that DKK1 in patients with periodontitis, rheumatoid patients, and acute infections is higher than that in normal groups. ${ }^{14,24}$
The increased levels of DKK1 in these inflammatory diseases are contrary to our findings, and it is clear that these results above cannot be completely explained by DKK1 being an inhibitor of canonical WNT/ $\beta$-catenin pathway. Atsushi Niida et al found that DKK1 may also be involved in the activation of the WNT pathway and that DKK1 mRNA increased when the WNT signal was activated using Wnt 3a or LiCl (an inhibitor of GSK3 $\beta$ ). ${ }^{25}$ DKK1 has also been observed to be associated with a $\beta$-cateninindependent WNT signal in Alzheimer's disease and tumor models, ${ }^{26-28}$ but the mechanism by which DKK1 activates the $\beta$-catenin-independent WNT signal is unclear, suggesting that the effects of DKK1 on cellular function are

Table 3 Multiple Linear Regression Analysis

\begin{tabular}{|l|l|l|l|l|l|}
\hline Parameter & R Square & Durbin-Watson & Unstandardized B & P-valve \\
\hline Age & 0.398 & 2.089 & & -17.807 & 0.293 \\
BMI & & & 95.256 & $0.038^{*}$ & 2.485 \\
FVC & & & -250.336 & 0.475 & 1.012 \\
FEVI & & & 775.820 & 6.034 \\
Sex & & & 435.982 & $0.016^{*}$ & 7.550 \\
Smoking history & & & 179.747 & 0.354 & 1.586 \\
\hline
\end{tabular}

Note: ${ }^{*}<<0.05$, the result is statistically significant. 

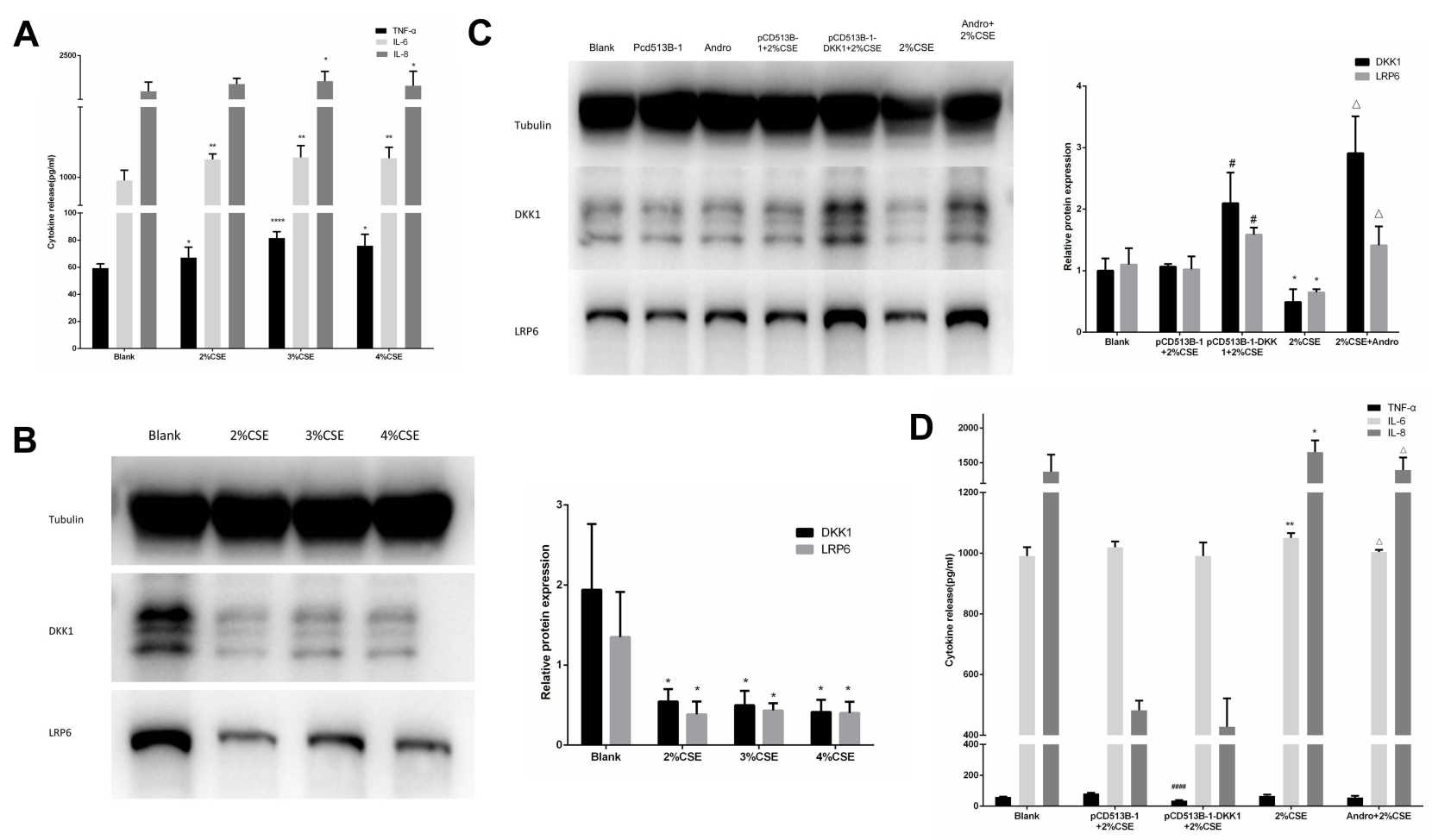

Figure 3 DKKI regulated LRP6 and airway inflammation, andrographolide elevated DKKI and LRP6 and reduced airway inflammation.

Notes: (A) Detection of TNF- $\alpha$, IL-6 and IL-8 in the cell supernatant after treatment of cells with different concentrations of CSE. (B) Western blot and PCR showed changes of DKKI and LRP6 after CSE treatment. (C) Cells were pre-transfected with DKKI overexpression plasmid or Andrographolide, followed by treatment with CSE, Western blot was used to detect protein levels. (D) Changes in TNF- $\alpha$, IL- 6 and IL-8 in cell supernatants in different treatment groups. Andro: Andrographolide. ${ }^{*} \mathrm{P}<0.05$ with, ${ }^{* *} \mathrm{p}<0.01$, ${ }^{* * * *} \mathrm{p}<0.000$ I, respect to the blank group, ${ }^{\#}<0.05,{ }^{\#} \mathrm{p}<0.000$ I, with respect to the CD 5 I $3 \mathrm{~B}-\mathrm{I}+2 \%$ CSE group, ${ }^{\Delta} \mathrm{p}<0.05$ with respect to the $2 \%$ CSE group.

multifaceted and DKK1 signaling in the lung may be highly complex. We expect that future studies will provide more insight into the role of DKK1 in the lungs.

In addition, our included COPD patients differed from controls in terms of age and smoking history, but the results of multiple linear regression analysis confirmed that age and smoking history had no effect on DKK1, and significant correlations were also found between DKK1 and FEV1, FEV1/FVC, and FEV1/Pre after correcting for age, sex, and smoking history.

In in vitro experiments, we found that IL-6, IL-8, and TNF- $\alpha$ increased and DKK1 decreased after airway epithelial cells treated with different concentrations of CSE. After overexpression of DKK1, the level of TNF- $\alpha$ in the cell supernatant was lower than that of the cigarette-induced group, which suggests that the reduction of DKK1 is involved in the regulation of cigarette-induced airway inflammation, and overexpression of DKK1 may be considered as a therapeutic approach to reduce airway inflammation. LRP6 is a receptor for DKK1 and previous studies have identified that the binding of LRP6 to DKK1 is involved in the regulation of inflammation, cell proliferation. ${ }^{7,29} \mathrm{We}$ found that when DKK1 increased, LRP6 also increased adaptively, suggesting that DKK1 can be involved in subsequent pathway regulation through upregulation and binding to LRP6, but the exact mechanism by which LRP6 is upregulated remains unclear and requires further study.

Previous studies confirmed that andrographolide reduced lung inflammation, Liao $\mathrm{W}$ et al found that andrographolide enhanced the anti-inflammatory effects of glucocorticoids in CS-exposed mice, ${ }^{30}$ Guan SP et al found that andrographolide also exerted antioxidant effects by directly reducing the number of inflammatory cells and enhancing Nrf2 activity in the lungs of CS-exposed mice. ${ }^{31}$ Our study provides a new hypothetical basis for the anti-inflammatory mechanism of andrographolide: andrographolide can ameliorate cigarette-dominated epithelial cell inflammation by upregulating DKK1 and increasing the binding of DKK1 to LRP6.

This study has some limitations, firstly, we combined COPD and AECOP patients in the specific analysis due to the small sample size. Secondly, this study lacks the relevant detection of WNT signaling pathway, and further mechanism research is expected to clarify the relationship 
between DKK1 and WNT in the pathogenesis of COPD. Moreover, there is no direct evidence that the combination of DKK1 and LRP6. The data in this study provide a new perspective on the anti-airway inflammation mechanism of andrographolide, which requires more in-depth study.

\section{Conclusion}

DKK1 is reduced in COPD and positively correlated with lung function, andrographolide and DKK1 can regulate the inflammation of airway epithelial cells induced by cigarette smoke extract by regulating LRP6. This not only indicates that DKK1 may be a biomarker of COPD, but also provides a new perspective on the role of DKK1 in COPD and andrographolide in reducing airway inflammation.

\section{Acknowledgments}

This research was funded by $1 \cdot 3 \cdot 5$ project for disciplines of excellence, West China Hospital, Sichuan University (ZYGD18006, ZYJC18012), National Natural Science Foundation of China (31871157, 81830001), and Sichuan Key Research and Development Program(2019YFS0232).

\section{Disclosure}

The authors report no conflicts of interest in this work.

\section{References}

1. Glinka A, Wu W, Delius H, Monaghan AP, Blumenstock C, Niehrs C. Dickkopf-1 is a member of a new family of secreted proteins and functions in head induction. Nature. 1998;391(6665):357-362. doi:10. $1038 / 34848$

2. Ren C, Gu X, Li H, et al. The role of DKK1 in Alzheimer's disease: a potential intervention point of brain damage prevention? Pharmacol Res. 2019;144:331-335. doi:10.1016/j.phrs.2019.04.033

3. Schunk SJ, Floege J, Fliser D, Speer T. WNT- $\beta$-catenin signalling a versatile player in kidney injury and repair. Nat Rev Nephrol. 2021;17(3):172-184. doi:10.1038/s41581-020-00343-w

4. Niehrs C. Function and biological roles of the Dickkopf family of Wnt modulators. Oncogene. 2006;25(57):7469-7481. doi:10.1038/sj.onc.12 10054

5. Chen L, Wang K, Shao Y, et al. Structural insight into the mechanisms of Wnt signaling antagonism by Dkk. J Biol Chem. 2008;283 (34):23364-23370. doi:10.1074/jbc.M802375200

6. Reinhold S, Blankesteijn WM. Wnt/B-Catenin Inhibitor Dickkopf 1. Arterioscler Thromb Vasc Biol. 2019;39(2):121-123. doi:10.1161/ ATVBAHA.118.312144

7. Gay A, Towler DA. Wnt signaling in cardiovascular disease: opportunities and challenges. Curr Opin Lipidol. 2017;28(5):387-396. doi:10.1097/MOL.0000000000000445

8. Baarsma HA, Königshoff M. 'WNT-er is coming': WNT signalling in chronic lung diseases. Thorax. 2017;72(8):746-759. doi:10.1136/thoraxjnl-2016-209753

9. Kim HT, Yin W, Nakamichi Y, et al. WNT/RYK signaling restricts goblet cell differentiation during lung development and repair. Proc Natl Acad Sci U S A. 2019;116(51):25697-25706. doi:10.1073/ pnas.1911071116
10. Vladar EK, Königshoff M. Noncanonical Wnt planar cell polarity signaling in lung development and disease. Biochem Soc Trans. 2020;48(1):231-243. doi:10.1042/BST20190597

11. Kneidinger N, Yildirim A, Callegari J, et al. Activation of the WNT/ $\beta$-catenin pathway attenuates experimental emphysema. Am J Respir Crit Care Med. 2011;183(6):723-733. doi:10.1164/rccm.200910$1560 \mathrm{OC}$

12. Cui W, Zhang Z, Zhang P, et al. Nrf2 attenuates inflammatory response in COPD/emphysema: crosstalk with $\mathrm{Wnt} 3 \mathrm{a} / \beta$-catenin and AMPK pathways. $J$ Cell Mol Med. 2018;22(7):3514-3525. doi:10.1111/jcmm.13628

13. Baarsma HA, Meurs H, Halayko AJ, et al. Glycogen synthase kinase3 regulates cigarette smoke extract- and IL-1 $\beta$-induced cytokine secretion by airway smooth muscle. Am J Physiol Lung Cell Mol Physiol. 2011;300(6):L910-919. doi:10.1152/ajplung.00232.2010

14. Mazon M, Larouche V, St-Louis M, Schindler D, Carreau M. Elevated blood levels of Dickkopf-1 are associated with acute infections. Immun inflammation dis. 2018;6(4):428-434. doi:10.1002/iid3.232

15. Klavdianou K, Liossis SN, Sakkas L, Daoussis D. The role of Dickkopf-1 in joint remodeling and fibrosis: a link connecting spondyloarthropathies and scleroderma? Semin Arthritis Rheum. 2017;46 (4):430-438. doi:10.1016/j.semarthrit.2016.08.014

16. Pfaff EM, Becker S, Günther A, Königshoff M. Dickkopf proteins influence lung epithelial cell proliferation in idiopathic pulmonary fibrosis. Eur Respir J. 2011;37(1):79-87. doi:10.1183/09031936.00142409

17. Guo Y, Mishra A, Howland E, et al. Platelet-derived Wnt antagonist Dickkopf-1 is implicated in ICAM-1/VCAM-1-mediated neutrophilic acute lung inflammation. Blood. 2015;126(19):2220-2229. doi:10.11 82/blood-2015-02-622233

18. Wang M, Zhang Y, Xu M, et al. Roles of TRPA1 and TRPV1 in cigarette smoke -induced airway epithelial cell injury model. Free Radic Biol Med. 2019;134:229-238. doi:10.1016/j.freeradbiomed.2019.01.004

19. Wang H, Yang T, Shen Y, et al. Ghrelin Inhibits Interleukin-6 Production Induced by Cigarette Smoke Extract in the Bronchial Epithelial Cell Via NF-kB Pathway. Inflammation. 2016;39 (1):190-198. doi:10.1007/s10753-015-0238-6

20. Duan H, Yan Z, Chen W, et al. TET1 inhibits EMT of ovarian cancer cells through activating Wnt/ $\beta$-catenin signaling inhibitors DKK1 and SFRP2. Gynecol Oncol. 2017;147(2):408-417. doi:10.1016/j.ygyno. 2017.08.010

21. Mao B, Wu W, Davidson G, et al. Kremen proteins are Dickkopf receptors that regulate Wnt/beta-catenin signalling. Nature. 2002;417 (6889):664-667. doi:10.1038/nature756

22. Voorzanger-Rousselot N, Goehrig D, Facon T, Clézardin P, Garnero P. Platelet is a major contributor to circulating levels of Dickkopf-1: clinical implications in patients with multiple myeloma. Br J Haematol. 2009;145(2):264-266. doi:10.1111/j.13 65-2141.2009.07587.x

23. Chae WJ, Ehrlich AK, Chan PY, et al. The Wnt Antagonist Dickkopf-1 Promotes Pathological Type 2 Cell-Mediated Inflammation. Immunity. 2016;44(2):246-258. doi:10.1016/j.immuni. 2016.01.008

24. Miranda TS, Napimoga MH, Feres M, et al. Antagonists of Wnt/ $\beta$ catenin signalling in the periodontitis associated with type 2 diabetes and smoking. J Clin Periodontol. 2018;45(3):293-302. doi:10.1111/ jcpe. 12854

25. Niida A, Hiroko T, Kasai M, et al. DKK1, a negative regulator of Wnt signaling, is a target of the beta-catenin/TCF pathway. Oncogene. 2004;23(52):8520-8526. doi:10.1038/sj.onc.1207892

26. Killick R, Ribe EM, Al-Shawi R, et al. Clusterin regulates $\beta$-amyloid toxicity via Dickkopf-1-driven induction of the wnt-PCP-JNK pathway. Mol Psychiatry. 2014;19(1):88-98. doi:10.1038/mp.2012.163

27. Thudi NK, Martin CK, Murahari S, et al. Dickkopf-1 (DKK-1) stimulated prostate cancer growth and metastasis and inhibited bone formation in osteoblastic bone metastases. Prostate. 2011;71 (6):615-625. doi:10.1002/pros.21277 
28. Wang S, Zhang S. Dickkopf-1 is frequently overexpressed in ovarian serous carcinoma and involved in tumor invasion. Clin Exp Metastasis. 2011;28(6):581-591. doi:10.1007/s10585-0119393-9

29. Sada R, Kimura H, Fukata Y, Fukata M, Yamamoto H, Kikuchi A. Dynamic palmitoylation controls the microdomain localization of the DKK1 receptors CKAP4 and LRP6. Sci Signal. 2019;12:608. doi:10.1126/scisignal.aat9519
30. Liao W, Lim AYH, Tan WSD, Abisheganaden J, Wong WSF. Restoration of HDAC2 and Nrf2 by andrographolide overcomes corticosteroid resistance in chronic obstructive pulmonary disease. Br J Pharmacol. 2020;177(16):3662-3673. doi:10.1111/bph.15080

31. Guan SP, Tee W, Ng DS, et al. Andrographolide protects against cigarette smoke-induced oxidative lung injury via augmentation of Nrf2 activity. Br J Pharmacol. 2013;168(7):1707-1718. doi:10.1111/ bph. 12054

\section{Publish your work in this journal}

The International Journal of COPD is an international, peer-reviewed journal of therapeutics and pharmacology focusing on concise rapid reporting of clinical studies and reviews in COPD. Special focus is given to the pathophysiological processes underlying the disease, intervention programs, patient focused education, and self management protocols. This journal is indexed on PubMed Central, MedLine and CAS. The manuscript management system is completely online and includes a very quick and fair peer-review system, which is all easy to use. Visit http://www.dovepress.com/testimonials.php to read real quotes from published authors. 